Cerebrovasc Dis 2007;24:540-542

DOI: $10.1159 / 000111220$

\section{Progressive Stroke Involving Bilateral Medial Medulla Expanding to Spinal Cord due to Vertebral Artery Dissection}

\author{
Noriko Hagiwara ${ }^{\text {a, }}$, Kazunori Toyoda ${ }^{\text {a }}$, Rina Torisu ${ }^{\text {a, b }}$, \\ Tooru Inoue ${ }^{\text {b }, K o t a r o ~ Y a s u m o r i ~}{ }^{\mathrm{c}}$, Setsuro Ibayashi ${ }^{\text {, }}$, \\ Yasushi Okada a
}

Departments of a Cerebrovascular Disease, ${ }^{\mathrm{b}}$ Neurosurgery and 'Neuroradiology, Cerebrovascular Center and Clinical Research Institute, National Hospital Organization Kyushu Medical Center, and ${ }^{\mathrm{d}}$ Department of Medicine and Clinical Science, Graduate School of Medical Sciences, Kyushu University, Fukuoka, Japan

Pain and numbness around the right posterolateral part of the neck developed in a 38-year-old Japanese man without vascular risk factors 2 weeks after carrying a child on his shoulders. Two weeks thereafter, vertigo and nausea developed, accompanied by a sharp pain in the right side of the neck, and he was quickly taken to a local hospital.

On the following day (day 1), quadriplegia developed immediately after anteflexion of the neck and the patient was transferred to our hospital. Upon admission, he was alert and well oriented. He had conjugated deviation with horizontal nystagmus on both lateral gazes, mild dysarthria and dysphagia. His tongue was normal. Quadriplegia was predominant on the left side. Deep tendon reflexes were increased and the Babinski reflexes were bilaterally positive. Touch, pain, and temperature sensations were diminished with marked hyperesthesia on both sides of the face and extremities. Joint and vibratory sensation was preserved. No other physical findings were remarkable. An MRI examination demonstrated a high intensity area in the lower medulla oblongata expanding to the upper cervical cord and axial images showed that the lesion involved the ventral half of the bilateral medial medulla oblongata and upper cervical cord, with partial dorsal extension (fig. 1A-F). The right vertebral artery (VA) was indistinct according to MR angiography (MRA) (fig. 1G, H). Doppler sonography revealed absent diastolic flow in the right VA, indicating occlusion of the right VA before branching of the posterior inferior cerebellar artery.

Based on MR and Doppler findings, he was diagnosed as having the bilateral medial medullocervical infarction due to right VA occlusion possibly due to dissection. He was continuously perfused with intravenous sodium heparin (10,000 units/day) together with intravenous glycerol and edaravone, a free radical scavenger. Intravenous methylprednisolone was administered for 3 days (total 1,200 mg) to suppress local cellular edema and save the microcirculation. His symptoms rapidly improved, and only mild left hemiparesis persisted by day 3 . Immediately after anteflexion of the neck on day 4 , however, he fell into a deep coma with complete quadriplegia and ataxic respiration requiring mechanical ventilation. Repeated MRI revealed no additional infarcts. Cerebral angiography showed that the right VA tapered off and finally occluded at the level of the foramen magnum. The sudden progression of the arterial occlusion suggested that dissection was the etiology. The anterior spinal artery (ASA) could not be identified. Fat-suppressed $\mathrm{T}_{1}$-weighted images showed a high crescent signal intensity surrounding the narrowed lumen of the right VA at the $\mathrm{C} 1-\mathrm{C} 2$ level (fig. $1 \mathrm{~F}$ ), suggesting an intraluminal hematoma due to dissection.

We immobilized his neck in a collar to prevent further progression of the arterial dissection by neck flexion. He regained consciousness and respiration recovered to normal on day 5. Quadriplegia and sensory deficits gradually improved and an MRA on day 29 showed that although stenotic and indistinct, the right VA was recanalized (fig. 1I, J). A Doppler sonogram on the same day showed that diastolic flow had recovered in the right VA. On day 77, he was discharged without need of living assistance, with the single residual effect being a neurogenic bladder.

\section{Discussion}

This patient was unique in two aspects. Firstly, unilateral VA dissection was the etiological mechanism of the bilateral medullocervical infarction. Secondly, anteflexion of the neck repeatedly triggered symptomatic deterioration probably by progressing the dissection.

Bilateral medial medullary infarction (MMI) might develop due to unilateral VA dissection from the point where an unpaired ASA branches off as an anatomical variation. However, systematic clinical studies have shown that of 12 patients with bilateral MMI among 126 with any MMI [1-7], only 1 patient developed stroke caused by unilateral VA dissection. The lower medulla that is usually supplied by the ASA was less the preferential site of MMI, and only 2 of the 12 patients with bilateral MMI described in previous reports had infarcts extending to the cervicomedullary junction, but not to the cervical cord $[1,5]$.

We applied anticoagulation to prevent the progression of brainstem ischemia under appropriate control of blood pressure and used radiological examinations to exclude aneurysmal changes in the cranial arteries. In addition, our experience revealed the importance of considering neck immobilization for acute stroke management due to VA dissection. Because the VA at the level of cervicomedullary junction is often compressed by neighboring structures [8], neck flexion and extension might mechanically stimulate a fragile artery in the absence of direct injury. Although the initial Doppler examination already indicated the VA occlusion, the neck anteflexion on day 4 might block the subtle anterograde flow via the narrow true lumen or the flow via the collat-

\section{KARGER}

Fax +41613061234 E-Mail karger@karger.ch www.karger.com (c) 2007 S. Karger AG, Basel

1015-9770/07/0246-0540\$23.50/0 

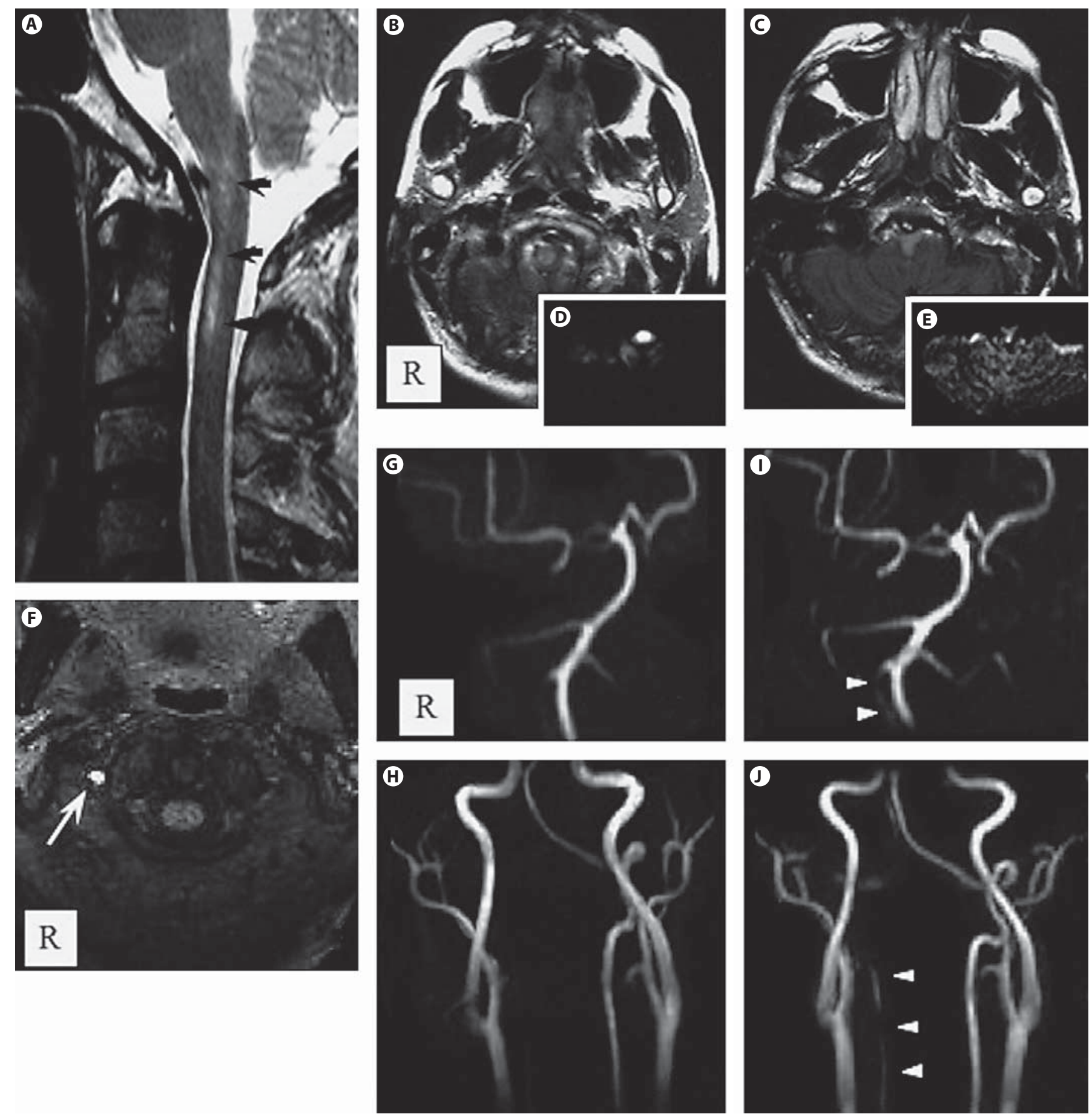

Fig. 1. Magnetic resonance images $(\mathbf{A}-\mathbf{F})$ and magnetic resonance angiography (G-J). $\mathbf{A} T_{2}$-weighted sagittal image shows high signal intensity at lower medulla expanding to upper cervical cord (arrows). $\mathrm{T}_{2}$-weighted axial image at the lower medulla (B) and C1 level (C) shows bilateral high intense lesions that also appeared as high signal intensity on diffusion-weighted images (D, E). F Fat-suppressed $\mathrm{T}_{1}$-weighted axial image on day 10 shows high crescent signal intensity surrounding narrow lumen of right vertebral artery (VA) at C1 level (arrow). At initial MRA, intracranial (G) and extracranial parts $(\mathbf{H})$ of right VA are hardly visible. Follow-up MRA on day 29 shows indistinct right VA (arrowheads) $(\mathbf{I}, \mathbf{J})$. 
eral circulation. Neck extension, in particular during sporting activities, is also known as a cause of the cervical carotid artery dissection [9]. Thus, a cervical collar might be indicated in patients with VA dissection whose stroke syndromes fluctuate.

\section{References}

1 Kim JS, Kim HG, Chung CS: Medial medullary syndrome report of 18 new patients and a review of the literature. Stroke 1995;26:1548-1552.

2 Toyoda K, Imamura T, Saku Y, Oita J, Ibayashi S, Minematsu K, Yamaguchi T, Fujishima M: Medial medullary infarction: analyses of eleven patients. Neurology 1996;47:1141-1147.

3 Bassetti C, Bogousslavsky J, Mattle H, Bernasconi A: Medial medullary stroke: report of seven patients and review of the literature. Neurology 1997;48:882-890.

4 Kim JS, Choi-Kwon S: Sensory sequelae of medullary infarction: differences between lateral and medial medullary syndrome. Stroke 1999; 30:2697-2703.

5 Kumral E, Afsar N, Kirbas D, Balkir K, Ozdemirkiran T: Spectrum of medial medullary infarction: clinical and magnetic resonance imaging findings. J Neurol 2002;249:85-93.
6 Kameda W, Kawanami T, Kurita K, Daimon M, Kayama T, Hosoya T, Kato T, for the Study Group of Association of Cerebrovascular Disease in Tohoku: Lateral and medial medullary infarction. A comparative analysis of 214 patients. Stroke 2004;35:694-699.

7 Kim JS, Choi KD, Oh SY, Park SH, Han MK, Yoon BW, Roh JK: Medial medullary infarction: abnormal ocular motor findings. Neurology 2005;65:1294-1298.

8 Schievink WI: Spontaneous dissection of the carotid and vertebral arteries. N Engl J Med 2001;344:898-906.

9 Lanczik O, Szabo K, Gass A, Hennerici MG: Tinnitus after cycling. Lancet 2003;362:292.

Noriko Hagiwara, MD

Department of Medicine and Clinical Science Graduate School of Medical Sciences, Kyushu University

Maidashi 3-1-1, Higashi-ku

Fukuoka 812-8582 (Japan)

Tel. +8192642 5256, Fax +81926425271

E-Mail hagiwara@intmed2.med.kyushu-u.ac.jp 